\title{
Quantum key distribution protocols using entangled state
}

\author{
Jian Wang * Quan Zhang, and Chao-jing Tang \\ School of Electronic Science and Engineering, \\ National University of Defense Technology, \\ Changsha, 410073, China
}

\begin{abstract}
We present three quantum key distribution protocols using entangled state. In the first two protocols, all Einstein-Podolsky-Rosen pairs are used to distribute a secret key except those chosen for eavesdropping check, because the communication parties measure each of their particles in an invariable measuring basis. The first protocol is based on the ideal of qubit transmission in blocks. Although it need quantum memory, its theoretic efficiency approximates to $100 \%$. The second protocol does not need quantum memory and its efficiency for qubits can achieve $100 \%$. In the third protocol, we present a controlled quantum key distribution using three-particle entangled state to solve a special cryptographic task. Only with the controller's permission could the communication parties establish their sharing key and the sharing key is secret to the controller. We also analyze the security and the efficiency of the present protocols.
\end{abstract}

PACS numbers: 03.67.Dd, 03.65.Ud

Quantum key distribution (QKD) is one of the most promising applications of quantum information science. The goal of QKD is to allow two legitimate parties, Alice and Bob, to generate a secret key over a long distance, in the presence of an eavesdropper, Eve, who interferes with the signals. The security of QKD is based on the fundamental laws of physics. Together with the Vernam cipher, QKD can be used for unconditionally secure communication. Since the BB84 protocol [1], the first QKD scheme, was published, many variations on QKD have been subsequently proposed. They can be roughly classified into "prepare and measure" protocols, such as BB84, B92 [2], the three-state protocol [3], the six-state protocol [4] and "entanglement based" protocols, such as E91 [5], BBM92 [6]. There have been efforts to set a security proof based on entanglement for the both classes [7]. Recently, the continuous-variable QKD $[8]$ has also been proved to be a promising protocol to send secret keys with high transmission rate.

The efficiency is one of the important parameters of QKD protocol. Many efforts have been made to improve the efficiency of QKD protocol. To improve the efficiency of the BB84 QKD, the scheme in Ref. [9] assigns significantly different probabilities for the different polarization bases during both transmission and reception to reduce the fraction of discarded data. The actual probabilities used in their schemes are announced in public. To defeat the eavesdropper's attack to the predominant basis, it needs a refined analysis of accepted data: they separate the accepted data into various subsets according to the basis employed and estimate an error rate for each subset individually. In Ref.[10], Hwang, Koh, and Han proposed a modified BB84 QKD scheme that increases its efficiency to nearly $100 \%$. However, the communication parties need a common secret key in their scheme.

*Electronic address: jwang@nudt.edu.cn
From the point of view of information theory, Cabello 11] defined the efficiency of a $\mathrm{QKD}$ protocol, $\mathcal{E}$,

$$
\mathcal{E}=\frac{b_{s}}{q_{t}+b_{t}}
$$

where $b_{s}$ is the number of secret bits received by Bob, $q_{t}, b_{t}$ is each the number of qubits, classical bits interchanged between Alice and Bob during the QKD process. Here the classical bits used for eavesdrop checking have been neglected. As has been discussed by Cabello, the efficiency of BB84, E91, cabello 2000 12, is 25\%, 50\%, $67 \%$, respectively. Actually, qubit is more expensive than classical bit. The efficiency equation $\mathcal{E}$ (called the total efficiency) cannot describe the efficiency of QKD protocol sufficiently. The efficiency for qubits is a useful complement to analyze the efficiency of QKD protocol, which is defined as

$$
\eta=\frac{q_{u}}{q_{t}}
$$

where $q_{u}$ is the useful qubits and $q_{t}$ is the total qubits transmitted [13]. To evaluate the efficiency of a QKD protocol, we should combine these two parameters.

In this paper, we present three QKD protocols using entangled state. The first protocol whose ideal is based on qubit transmission in blocks uses Einstein-PodolskyRosen (EPR) pairs to distribute a secret key. The theoretic efficiency of the protocol approximates to $100 \%$, because all EPR pairs are used to distribute a secret key except those chosen for checking eavesdroppers. However, the flaw of the first protocol is that it needs quantum memory during the QKD process. We then present the second protocol which does not need quantum memory. In the second protocol, although the total efficiency is $50 \%$, the efficiency for qubits approaches $100 \%$. To solve a special cryptographic task, we propose a controlled QKD protocol. The two communication parties can only generate their sharing key with the permission 
of the controller. This protocol can also be used to distribute a secret key among three parties. The three protocols are unconditionally secure.

G. L. Long and X. S. Liu proposed an efficient highcapacity QKD scheme 14] whose efficiency can achieve $100 \%$. In their scheme, a set of ordered $N$ EPR pairs is used as a data block for distributing secret key. Based on the ideal of block transmission, we present another QKD protocol whose efficiency can also achieve $100 \%$. The security of the present protocol is ensured by the random Hadamada transformation. Our protocol is as follows:

Protocol 1:

(1) Alice prepares an ordered $N$ EPR pairs in the Bell state

$$
\left|\phi^{+}\right\rangle_{A B}=\frac{1}{\sqrt{2}}(|00\rangle+|11\rangle)_{A B} .
$$

We denotes the ordered $N$ EPR pairs with $\left\{\left[\mathrm{P}_{1}(A), \mathrm{P}_{1}(B)\right],\left[\mathrm{P}_{2}(A), \mathrm{P}_{2}(B)\right], \cdots,\left[\mathrm{P}_{N}(A), \mathrm{P}_{N}(B)\right]\right\}$, where the subscript indicates the pair order in the sequence, and $A, B$ represents the two particles of EPR pair, respectively. Alice takes one particle from each EPR pair to form an ordered EPR partner particle sequence $\left[\mathrm{P}_{1}(A), \mathrm{P}_{2}(A), \cdots, \mathrm{P}_{N}(A)\right]$, called $A$ sequence. The remaining EPR partner particles compose $B$ sequence, $\left[\mathrm{P}_{1}(B), \mathrm{P}_{2}(B), \cdots, \mathrm{P}_{N}(B)\right]$. Alice transmits the $B$ sequence to Bob.

(2) To prevent eavesdropping, Bob selects randomly a sufficiently large subset of $B$ sequence and performs Hadamard transformations on them. He then announces publicly the position of the selected particles. The Hadamard transformation is crucial for the security of the scheme as we will see in the sequel.

(3) After hearing from Bob, Alice executes Hadamard transformations on the corresponding particles of $A$ sequence.

(4) Note that

$$
\left|\phi^{+}\right\rangle_{A B}=\frac{1}{\sqrt{2}}(|++\rangle+|--\rangle)_{A B},
$$

where $|+\rangle=\frac{1}{\sqrt{2}}(|0\rangle+|1\rangle),|-\rangle=\frac{1}{\sqrt{2}}(|0\rangle-|1\rangle)$. The two parties' Hadamada transformations will not change the initial state. Alice then measures the $A$ sequence in $Z$-basis $\{|0\rangle,|1\rangle\}$. Bob measures the $B$ sequence in the same basis as Alice. Thus they established sharing key. In this step, they can also measure each of their particles in $X$-basis $\{|+\rangle,|-\rangle\}$.

(5) Alice and Bob then publicly compare the results of these measurements to check eavesdropping. Bob chooses randomly a sufficiently large subset of his results and announces them publicly. Alice compares Bob's results with her corresponding results. She can thus find out whether there is an eavesdropper. If too many of these measurements disagree, they abort the protocol. If they are certain that there is no eavesdropping, Alice and Bob utilize privacy amplification and error correction to distil the final key.
We now discuss the security and the efficiency of protocol 1. The crucial point is that the Hadamard transformations at the step 2 and 3 of the protocol do not allow Eve to have a successful attack and Eve's attack will be detected during the eavesdropping check. The protocol 1 is similar to the modified Lo-Chau protocol [15], but the protocol 1 does not need classical message except that used for eavesdropping check. The security of the protocol 1 is the same as the modified Lo-Chau protocol which is proved unconditionally secure. According to the information-theoretic efficiency defined by Cabello, the total efficiency of protocol 1 can be made asymptotically close to $100 \%$. Here the classical bits used for eavesdrop checking have been neglected. The efficiency for qubit can also achieve $100 \%$ because all EPR pairs are used to distribute a sharing key except those used to check eavesdropping.

Although the protocol 1 is efficient, it is necessary for the protocol to use quantum memory. We then present another QKD protocol without quantum memory.

Protocol 2:

(1) Alice prepares randomly an EPR pair in one of the following states

$$
\left|\phi^{ \pm}\right\rangle_{A B}=\frac{1}{\sqrt{2}}(|00\rangle \pm|11\rangle)_{A B},
$$

where the subscripts $A, B$ represents the two particles of EPR pairs. Alice then send the particle $B$ to Bob.

(2) After hearing from Bob, Alice publishes the information of the initial state she prepared. If the initial state is $\left|\phi^{-}\right\rangle$, both Alice and Bob perform Hadamard transformation on each of their corresponding particle. Otherwise, they do nothing. After Hadamada transformation, $\left|\phi^{-}\right\rangle$is changed to

$$
\left|\phi_{1}^{-}\right\rangle_{A B}=\frac{1}{\sqrt{2}}(|++\rangle-|--\rangle)_{A B},
$$

(3) After doing this, Alice and Bob measure their corresponding particles in $X$-basis. They agree that $|+\rangle$ $(|-\rangle)$ corresponds to bit "0" (" 1 ").

(4) The parties repeat the above steps $N$ times and generate $N$ raw secret keys.

(5) To check eavesdropping, Alice selects randomly a sufficiently large subset of her measurement results and tells it to Bob. Bob publishes his measurement results of the sampling particles. Alice then evaluates the error rate of the QKD process. If the error rate exceeds the threshold, they abort the protocol. Otherwise, they utilize privacy amplification and error correction to distil a final key.

We then analyze the security and the efficiency of protocol 2. Firstly, the protocol is secure against the intercept-resend attack by Eve. In this attack, Eve intercepts the particle $B$ and makes measurement on it, then she resends a particle to Bob according to her measurement result. Eve can only intercept the particle $B$ at the step 1 of the protocol and she cannot make certain 
which particle will be executed Hadamard transformation. Thus Eve can only measure the intercepted particle in $Z$-basis or $X$-basis randomly. Suppose Eve measures the intercepted particle which belongs to $\left|\phi^{+}\right\rangle$in $Z$-basis. If the result of Eve's measurement is " 0 ", she sends a particle in the state $|+\rangle$ to Bob, otherwise sends a particle in the state $|-\rangle$. Then the state of the two particles collapses to $|0+\rangle_{A B}$ or $|1-\rangle_{A B}$ each with probability $1 / 2$. Thus the error rate introduced by Eve will achieve $50 \%$. During the eavesdropping check, Eve's attack will be detected. Suppose Eve performs $X$-basis measurement on the intercepted particle which belongs to $\left|\phi^{-}\right\rangle$. Then the state of the two particles collapses to $|+-\rangle_{A B}$ or $|-+\rangle_{A B}$ each with probability $1 / 2$. After Hadamada transformation, the state is changed to $|01\rangle_{A B}$ or $|10\rangle_{A B}$. The error rate introduced by Eve will also achieve $50 \%$.

Secondly, the protocol is safe against collective attack. In this strategy, Eve intercepts the particle $B$ and uses it and her own ancillary particle in the state $|0\rangle$ to do a CNOT operation (the particle $B$ is the controller, Eve's ancillary particle is the target). Then Eve resends the particle $B$ to Bob. However, Eve has no information of the initial state. Suppose the initial state is

$$
\left|\phi^{-}\right\rangle_{A B}=\frac{1}{\sqrt{2}}(|+-\rangle+|-+\rangle)_{A B} .
$$

After Eve's collective attack, the state of the particle $A$, $B$ and Eve's ancillary particle becomes

$$
\left|\Omega_{1}\right\rangle_{A B E}=\frac{1}{\sqrt{2}}(|+-1\rangle+|-+0\rangle)_{A B E},
$$

where the subscript $E$ indicates Eve's ancillary particle. According to the protocol, Alice and Bob perform Hadamada transformations on their corresponding particles, obtaining

$$
\left|\Omega_{2}\right\rangle_{A B E}=\frac{1}{\sqrt{2}}(|011\rangle+|100\rangle)_{A B E} .
$$

Obviously, Eve's eavesdropping will be detected during the eavesdropping check because half of Bob's results will be inconsistent with that of Alice's. As we described above, the total error rate introduced by Eve is $25 \%$. Actually, the security of the protocol 2 is equal to that of the modified Lo-Chau protocol. We can also give a Shor-Preskill-type proof to protocol 2.

In the protocol 2, the efficiency for qubits is $100 \%$ because all EPR pairs are used to generate a key except those chosen for eavesdropping check. It is not necessary for the protocol 2 to use quantum memory. However, it needs a bit of classical message to generate a bit of sharing key. Therefor the total efficiency of the protocol 2 is $50 \%$. Certainly, we should pay more attention to the efficiency for qubits because qubit is more expensive than classical bit.

Han et al. proposed a controlled QKD scheme with three-particle entanglement [16]. According to their scheme, the Eq. 1 of their scheme should be

$$
\begin{aligned}
|A B C\rangle= & \frac{1}{2}(|H H H\rangle+|H V V\rangle+|V H V\rangle-|V V H\rangle) \\
= & \frac{1}{2}[|H\rangle(|H H\rangle+|V V\rangle) \\
& +|V\rangle(|H V\rangle-|V H\rangle)]
\end{aligned}
$$

Suppose Bob intercepts the two photons which Alice sends to Bob and Carol. He then performs Bell basis measurement on the intercepted photons and obtains $\left|\phi^{+}\right\rangle$and $\left|\psi^{-}\right\rangle$each with probability $1 / 2$. Bob will obtain Alice's measurement result and his action will not be detected by Alice. Bob then resends one of the intercepted particles to Carol. According to their scheme, Bob and Carol can establish sharing key without the control of Alice. To solve this problem, we propose another controlled QKD protocol. Suppose only Alice can prepare the entangled state. Bob and Charlie can only generate their sharing key under the control of the controller Alice. Without Alice's permission, Bob and Charlie cannot establish their sharing key. Certainly, the sharing key is secret to Alice.

\section{Protocol 3:}

(1) Alice prepares a three-particle entangled state in the state

$$
\left|\Psi_{1}\right\rangle_{A B C}=\frac{1}{\sqrt{2}}(|0+0\rangle+|1-1\rangle)_{A B C}
$$

or

$$
\left|\Psi_{2}\right\rangle_{A B C}=\frac{1}{\sqrt{2}}(|0-0\rangle+|1+1\rangle)_{A B C}
$$

randomly. She then sends the particle $B, C$ to Bob and Charlie, respectively.

(2) After confirming that Bob and Charlie have received their particles, Alice measures the particle $A$ in $Z$ basis or $X$-basis randomly. She then publishes his measuring basis. If Alice performed $Z$-basis measurement, Bob measures the particle $B$ in the $X$-basis and Charlie performs $Z$-basis measurement on the particle $C$. If Alice performs $X$-basis measurement, Bob (Charlie) performs $Z$-basis ( $X$-basis) measurement on his particle.

(3) The parties repeat the above steps $N$ times and each obtains $N$ measurement results. The value of $N$ is large enough to check eavesdropping, but not enough to generate a key which is long enough to ensure the security of cryptography. To generate a secure key, the parties should run the protocol twice at least.

(4) Alice chooses randomly a sufficiently large subset of her measurement results and announces the positions of the sampling particles publicly. She let Bob and Charlie publish their measurement results of the sampling particles. Note that

$$
\begin{aligned}
\left|\Psi_{1}\right\rangle_{A B C}= & \frac{1}{\sqrt{2}}[|+\rangle(|0+\rangle+|1-\rangle) \\
& +|-\rangle(|0-\rangle+|1+\rangle)]_{A B C}
\end{aligned}
$$


and

$$
\begin{aligned}
\left|\Psi_{2}\right\rangle_{A B C}= & \frac{1}{\sqrt{2}}[|+\rangle(|0+\rangle-|1-\rangle) \\
& +|-\rangle(|0-\rangle-|1+\rangle)]_{A B C} .
\end{aligned}
$$

They can make certain whether there exists eavesdropping by comparing their measurement results. If Bob performs Bell basis measurement on the particle $B$ and $C$, his action will also be detected by Alice. If there is no eavesdropping, they continue to the next step. Otherwise, the protocol is halted.

(5) If Alice permits Bob and Charlie to establish their sharing key, she publishes her measurement results of the particles on which she performed $X$-basis measurements. The parties let $|0\rangle,|+\rangle$ correspond to binary "0" and $|1\rangle$, $|-\rangle$ correspond to binary "1". If Alice's measurement result is $|+\rangle$, Bob and Charlie obtains a identical raw key "0" or " 1 ". If Alice's measurement result is $|-\rangle$, Bob or Charlie should invert the bit value of the key to obtain a identical key. Obviously, the key is secret to the controller Alice. If Alice, Bob and Charlie want to establish a three-party key, it only needs Alice to publish the initial state of the particles on which Alice performed $Z$-basis measurement. If the initial state is $\left|\Psi_{2}\right\rangle$, Bob should invert his bit of the key. They can also distil a final key using privacy amplification and error correction.

We now discuss the security of the protocol 3. The two state $\left|\Psi_{1}\right\rangle,\left|\Psi_{2}\right\rangle$ which are prepared randomly by Alice, ensure that Bob and Charlie cannot establish sharing key without Alice's permission. Suppose Bob intercepts the particle $B$ and $C$ and performs Bell basis measurement on the intercepted particles. Note that

$$
\begin{aligned}
|0+\rangle+|1-\rangle & =\frac{1}{\sqrt{2}}(|00\rangle-|11\rangle+|01\rangle+|10\rangle) \\
|0-\rangle+|1+\rangle & =\frac{1}{\sqrt{2}}(|00\rangle+|11\rangle-|01\rangle+|10\rangle) \\
|0+\rangle-|1-\rangle & =\frac{1}{\sqrt{2}}(|00\rangle+|11\rangle+|01\rangle-|10\rangle) \\
|0-\rangle-|1+\rangle & =\frac{1}{\sqrt{2}}(|00\rangle-|11\rangle-|01\rangle-|10\rangle) .
\end{aligned}
$$

According to the Eq. [13]and 14] Bob cannot make certain the state of the particle $B, C$. He also has no information of Alice's result. During the eavesdropping check, his action will be detected by Alice. Alice then stops the protocol. Because the parties have run the protocol only once, Bob and Charlie cannot generate a secure sharing key even if they obtained some random EPR pairs. Without regard to the special cryptographic task, the security of the present protocol can be reduced to that of the BBM92 protocol because the parties measure their corresponding particles in $Z$-basis or $X$-basis randomly.

To improve the efficiency of the protocol 3, we can use the same method of the Ref. [16], which Alice measures her particle in the $Z$-basis ( $X$-basis) with probabilities $\varepsilon$ $(1-\varepsilon)$, where $0<\varepsilon \leq 1$. Obviously, it needs a refined data analysis to ensure the security of the protocol.

In summary, we propose three QKD protocols with entangled state. It is not necessary for the protocol 1 and protocol 2 to use alternative measuring basis. Thus all EPR pairs of the two protocols are used to generate a secret key except those chosen for eavesdropping. By using block transmission, the total efficiency of the protocol 1 can achieve $100 \%$ but it needs quantum memory. The protocol 2 does not need quantum memory and the efficiency for qubits is $100 \%$ because the parties measure their particles in an invariable measuring basis. But the total efficiency of the protocol 2 is $50 \%$ for the use of classical bit. In view of the fact that qubit is more expensive than classical bit, more attention should be paid to the efficiency for qubits. The protocol 3 is a controlled QKD protocol which can be applied to a special cryptographic task. Bob and Charlie establish their sharing key under the control of Alice. Only with Alice's permission could Bob and Charlie generate an identical key. The protocol 3 is multifunctional, which can also be used to distribute a key among three parties. It is appropriate for QKD network.

This work is supported by the National Natural Science Foundation of China under Grant No. 60472032.
[1] C. H. Bennett and G. Brassard, in Proceedings of IEEE international Conference on Computers, Systems and signal Processing, Bangalore, India (IEEE, New York), pp. 175 - 179 (1984).

[2] C. H. Bennett, Phys. Rev. Lett. 68, 3121 (1992).

[3] H. Bechmann-Pasquinucci and A. Peres, Phys. Rev. Lett. 85, 3313 (2000).

[4] D. Bruss, Phys. Rev. Lett. 81, 3018 (1998).

[5] A. K. Ekert, Phys. Rev. Lett. 67, 661 (1991).

[6] C. H. Bennett, G. Brassard and N. D. Mermin, Phys. Rev. Lett. 68, 557 (1992).

[7] M. Curty, M. Lewenstein, and N. Lutkenhaus, Phys. Rev. Lett. 92, 217903 (2004).
[8] F. Grosshans, G. Van Assche, J.Wenger, R. Brouri, N. J. Cerf, and P. Grangier, Nature (London) 421, 238 (2003).

[9] H. K. Lo, H. F. Chau, M. Ardehali, Journal of Cryptology 18, 133 (2004)

[10] W. Y. Hwang, I. G. Koh, and Y. D. Han, Phys. Lett. A 244, 489 (1998)

[11] A. Cabello, Phys. Rev. Lett. 85, 5635 (2000).

[12] A. Cabello, Phys. Rev. A 61, 052312 (2000).

[13] F. G. Deng et al., quant-ph/0508118, (2005).

[14] G. L. Long and X. S. Liu, Phys. Rev. A 65, 032302 (2002).

[15] P. W. Shor and J. Preskill, Phys. Rev. Lett. 85, 441 (2000). 
[16] C. Han, P. Xue, G. C. Guo, Chin. Phys. Lett. 20, 183, (2003). 